\section{Delayed pneumothorax after CT-guided percutaneous fine needle aspiration lung biopsy}

\author{
Zoë C Traill, Fergus V Gleeson
}

\begin{abstract}
Two patients are described who developed pneumothoraces more than 24 hours after computed tomography (CT) guided percutaneous fine needle aspiration lung biopsies. The pneumothoraces required treatment in both cases. Such delayed pneumothorax after lung biopsy is extremely unusual. Patients should be warned of the possible occurrence of this complication and instructed to seek medical help if they develop chest pain or breathlessness.
\end{abstract}

(Thorax 1997;52:581-582)

Keywords: complications, lung biopsy, pneumothorax.

Percutaneous fine needle aspiration biopsy is a well established method for diagnosing lung lesions. ${ }^{1-3}$ Although generally a safe and well tolerated procedure, pneumothorax is a relatively common and potentially serious complication. ${ }^{45}$ We describe two patients who developed significant pneumothoraces more than 24 hours after percutaneous fine needle aspiration lung biopsy.

\section{Case reports}

CASE 1

A 56 year old woman presented with a one month history of right sided Jacksonian-type seizures. She was a life long smoker with no past medical history of note. Physical examination was unremarkable. A computed tomographic (CT) scan of the brain revealed a single enhancing lesion in the left hemisphere with surrounding oedema in keeping with a metastasis. A chest radiograph showed a small mass in the left upper lobe. A chest CT scan confirmed the presence of a $2 \mathrm{~cm}$ mass in the left upper lobe adjacent to the aortic arch which was thought most likely to represent a primary bronchial carcinoma. A fine needle (22 gauge) aspiration biopsy specimen of the left upper lobe mass was taken percutaneously under CT guidance and revealed adenocarcinoma cells. No pneumothorax was seen on the CT scan performed immediately after the procedure, nor on chest radiographs taken at one and four hours after the procedure. The patient was discharged home the same day, but re-presented 26 hours after the lung biopsy with a sudden onset of left chest pain associated with minor exertional dyspnoea. A chest radiograph at this time showed a moderate left pneumothorax which was aspirated with good effect. Over the subsequent months she received symptomatic benefit from cranial radiotherapy, dexamethasone, and anticonvulsants but progressively deteriorated and died 10 months after the start of her illness.

CASE 2

A 68 year old male smoker presented with a one week history of intermittent claudication of his right leg. A chest radiograph performed as part of his routine assessment showed enlarged right paratracheal nodes and a $3 \mathrm{~cm}$ lobulated mass in the right upper lobe. A chest CT scan confirmed the presence of a mass within the anterior segment of the right upper lobe and large volume mediastinal and right hilar lymphadenopathy. Bronchoscopic examination was normal and cytological examination of bronchial brushings and washings did not show malignant cells. CT guided percutaneous fine needle (22 gauge) aspiration biopsy of the mass revealed small cell lung carcinoma. A CT scan taken after the procedure did not show a pneumothorax, neither did chest radiographs taken at one and four hours after the biopsy. He was discharged home the same day. Approximately 36 hours after the biopsy he became acutely breathless but did not seek medical attention. His dyspnoea improved over the next 48 hours but then worsened and he was re-admitted four days after the biopsy. A chest radiograph showed a right pneumothorax which was successfully managed by intercostal chest drain insertion. He is currently well and undergoing chemotherapy.

\section{Discussion}

Radiologically guided percutaneous fine needle aspiration biopsy is a well established technique in the diagnosis of lung lesions. Sensitivities in the detection of malignancy in excess of $90 \%$ are repeatedly obtained..$^{23}$ It is routinely performed as an outpatient procedure. ${ }^{5}$ Pneumothorax is the most common and potentially serious complication. Rates of pneumothorax of about $25 \%$ are common ${ }^{67}$ although most do not require treatment. In a study of 673 patients in whom transthoracic fine needle aspiration biopsy was performed Perlmutt and colleagues ${ }^{6}$ did not have a single case of pneumothorax occurring more than four hours after the procedure. They recommended that a chest radiograph should be taken at one and four hours after such biopsies in outpatients.

In our institution CT guidance is preferred over fluoroscopic guidance in percutaneous transthoracic needle biopsy for those lesions which are poorly seen or considered inaccessible at fluoroscopy and for lesions adjacent to major cardiovascular structures, either hilar or mediastinal. The pneumothorax rate appears to be higher with CT guidance than that commonly reported with fluoroscopy. ${ }^{8}$ This is likely to be due to the increased time 
that the biopsy needle remains in the lung, although the increased sensitivity of CT scanning compared with chest radiography in the detection of pneumothorax must be contributory. The two patients we describe both had CT scans performed at the level at which the needle crossed the pleural surface immediately after the biopsy. In neither of these was a pneumothorax apparent. Their delayed pneumothoraces were not therefore due to enlargement of an initial pneumothorax not visible on chest radiographs taken one and four hours after the procedure. Although neither of our two patients gave a history of sudden exertion or coughing prior to the onset of symptoms, it may be that their delayed pneumothoraces were secondary to displacement of small pleural blood clots following the biopsy procedure.

Delayed pneumothorax (of more than 24 hours) has been reported as a complication of transbronchial lung biopsy ${ }^{9}$ and also of subclavian vein catheterisation. ${ }^{10}$ The occurrence of pneumothorax in two patients more than 24 hours after the procedure emphasises the importance of considering this diagnosis even at a delayed stage. Every patient who undergoes this procedure should be warned of the importance of seeking medical attention should they develop increased breathlessness or chest pain after discharge.

1 Thornbury JR, Burke DP, Naylor B. Transthoracic needle aspiration biopsy: accuracy of cytologic typing of malignant neoplasms. AfR 1981;136:719-24.

2 Khouri NF, Stitik FP, Erozan YS, Gupta PK, Kim WS Scott WW, et al. Transthoracic needle aspiration biopsy of benign and malignant lung lesions. AfR 1985;144 $281-8$

3 Stanley JH, Fish GD, Andriole JG, Gobien RP, Betsill WL, Laden SA, et al. Lung lesions: cytologic diagnosis by fineneedle biopsy. Radiology 1987;162:389-91.

4 Westcott JL. Direct percutaneous needle aspiration of localized pulmonary lesions: results in 422 patients. Radiology 1980;137:31-5.

5 Stevens GM, Jackman RJ. Outpatient needle biopsy of the lung: its safety and utility. Radiology 1984;151:301-4.

6 Perlmutt LM, Braun SD, Newman GE, Oke EJ, Dunnick NR. Timing of chest film follow-up after transthoracic needle aspiration. AfR 1986;146:1049-50.

7 Moore EH, Shepard JO, McLoud TC, Templeton PA, Kosuik JP. Positional precautions in needle aspiration lung biopsy. Radiology 1990;175:733-5.

8 van Sonnenberg E, Casola G, Ho M, Neff CC, Varney RR, Wittich GR, et al. Difficult thoracic lesions: CT-guided biopsy experience in 150 cases. Radiology 1988;167:45761.

9 Levy H, Kallenbach JM, Feldman C, Pincus P, Hurwitz M Delayed pneumothorax after transbronchial lung biopsy. Thorax 1986;41:647-8.

10 Spiliotis J, Kordossis T, Kalfarentzos F. The incidence of delayed pneumothorax as a complication of subclavian vein catheterisation. Br f Clin Pharmacol 1992;46:171-2.

\section{Neuromuscular blockade with acute respiratory failure in a patient receiving cibenzoline}

Thomas Similowski, Christian Straus, Valérie Attali, Frédéric Girard, François Philippe, Gilbert Deray, Daniel Thomas, Jean-Philippe Derenne

Pneumologie et Réanimation \&

Laboratoire de

Physiopathologie

Respiratoire

T Similowski

C Straus

V Attali

F Girard

J-P Derenne

Service de Cardiologie

F Philippe

D Thomas

Service de Néphrologie G Deray

Groupe Hospitalier Pitié-Salpêtrière, 75651 Paris Cedex 13, France

Correspondence to: Dr T Similowski.

Received 27 January 1996 Returned to authors 4 April 1996

Revised version received

7 June 1996

Accepted for publication

18 July 1996

\begin{abstract}
Cibenzoline is a class Ic antiarrhythmic agent that can be used to treat supraventricular arrhythmias. A case is reported of cibenzoline overdose in a patient with impaired renal function, leading not only to the usual cardiac and metabolic symptoms (bradycardia and hypoglycaemia), but also to a myastheniform syndrome with acute respiratory failure. Neuromuscular blockade was demonstrated by repetitive supramaximal stimulation of the median nerve, and diaphragmatic involvement was evidenced by applying the same protocol to the phrenic nerve. Muscle strength recovered as serum cibenzoline levels decreased, allowing the patient to be weaned from the ventilator. This observation suggests that cibenzoline, like other antiarrhythmic agents,
\end{abstract}

can be responsible for neuromuscular blockade, and should therefore be used with caution in patients with neuromuscular and respiratory diseases or with impaired renal function.

(Thorax 1997;52:582-584)

Keywords: cibenzoline, neuromuscular blockade, acute respiratory failure, diaphragm paralysis, phrenic nerve stimulation.

A host of drugs can interfere with the contraction of respiratory muscle at several levels ${ }^{1}$ including impairment of neuromuscular transmission. The latter can result in life threatening episodes of respiratory failure, ${ }^{2}$ particularly in patients with pre-existing myasthenia gravis and other neuromuscular diseases, and in patients with a reduced respiratory reserve or a decreased ability to eliminate drugs. ${ }^{12}$

We report a case of acute respiratory failure with electrophysiological evidence of neuromuscular blockade involving the diaphragm in a patient with chronic renal failure receiving cibenzoline, a class Ic antiarrhythmic agent. ${ }^{3}$ This case appears to be the first report of cibenzoline-induced neuromuscular blockade. The initial report of cibenzoline-induced hypoglycaemia ${ }^{4}$ mentions the association of bradycardia, central nervous system depression, and respiratory distress but no details are given.

\section{Case report}

CLINICAL HISTORY

The patient was a 75 year old man with end stage chronic renal failure from polycystic renal disease, undergoing continuous ambulatory peritoneal dialysis (CAPD) and receiving oral 

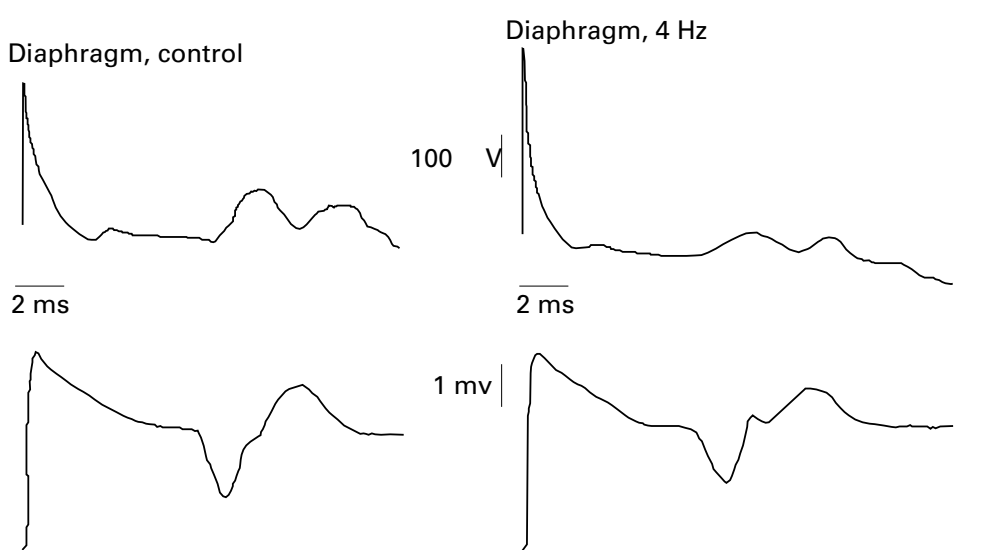

Abductor pollicis brevis, control

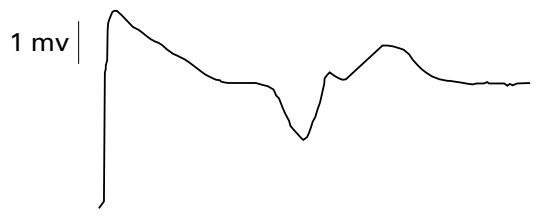

Abductor pollicis brevis, $4 \mathrm{~Hz}$

Figure 1 Examples of action potentials of the right diaphragm and abductor pollicis brevis muscle in response to supramaximal transcutaneous stimulation of the right phrenic and median nerves, respectively. The left tracings correspond to an isolated single shock and the right tracings were obtained following several repeated shocks delivered at a frequency of $4 \mathrm{~Hz}$. There was a marked decrease in the peak-to-peak amplitude of the response observed in the two conditions of stimulation, strongly suggesting neurotransmission failure. electrodes during repetitive transcutaneous supramaximal stimulation of the right phrenic $^{56}$ and median nerves, respectively (square wave $0.1 \mathrm{~ms}$ shocks delivered at $4 \mathrm{~Hz}$ for a few seconds; Neuropack Sigma, NihonKohden, Japan). The action potentials were measured in the seventh right intercostal space on the anterior axillary line for the diaphragm and over the muscle mass of the thenar eminence with reference to the first phalange of the thumb for the APB.

In both muscles the response to a single stimulus was normal in shape and latency (phrenic nerve conduction time $7.1 \mathrm{~ms}$ ), but a significant decrement in amplitude occurred after stimulation at $4 \mathrm{~Hz}(58 \%$ and $24 \%$ for the diaphragm and $\mathrm{APB}$, respectively; fig 1). Single shock-induced action potentials returned to their original amplitude a few seconds after discontinuing the sustained stimulation.

BIOLOGICAL AND CLINICAL COURSE

From day 7 to day 9 the patient remained weak and unable to inhale; diffuse fasciculations persisted but were less intense. On day 10 the patient began to trigger the ventilator when set to $-2 \mathrm{~cm} \mathrm{H}_{2} \mathrm{O}$. Fasciculations and ptosis had completely disappeared and, clinically, the strength of the limb muscles had noticeably increased. On day 11 the patient was able to generate intrathoracic pressures of $-15 \mathrm{~cm}$ $\mathrm{H}_{2} \mathrm{O}$ and sustained spontaneous ventilation for two hours. He was extubated on day 14 and discharged on day 16 .

Peritoneal dialysis was continuously maintained during the stay in the intensive care unit, uraemia remaining roughly constant (25-30 mmol/l, comparable to the patient's steady state). Cibenzoline levels decreased continuously from $2.2 \mathrm{mg} / 1$ on admission to 2.0 , $0.97,0.62,0.40$, and $0 \mathrm{mg} / \mathrm{l}$ on days $8,9,10$, 11 , and 12 , respectively.

Six months after the acute episode uraemia was unchanged. There was no evidence of residual muscle weakness, with spirometric values within the normal range and a mouth pressure response to cervical magnetic stimulation reaching $12.5 \mathrm{~cm} \mathrm{H}_{2} \mathrm{O}$.

\section{Discussion}

DIAGNOSIS OF NEUROMUSCULAR BLOCKADE AND OF DIAPHRAGMATIC INVOLVEMENT

The clinical picture observed during the first 24 hours in the respiratory intensive care unit was highly suggestive of impaired neuromuscular transmission. A test with an acetylcholinesterase inhibitor could have supported the diagnosis further. However, in the context of an underlying supraventricular arrhythmia in an elderly patient and of cibenzoline-induced bradycardia, cardiac risks potentially associated with edrophonium chloride (Tensilon) or prostigmine were considered too important, particularly since a negative test would not have ruled out neuromuscular blockade. Furthermore, the pattern of response to a standard electrophysiological detection protocol accorded well with the diagnosis (fig 1).
Diaphragm and abductor pollicis brevis (APB) action potentials were measured with surface 
Dysfunction of the respiratory muscles in situations where neuromuscular transmission is impaired is a well known fact, but is generally diagnosed solely on the basis of clinical features. To our knowledge, only one study by Mier $e t a l^{6}$ has specifically examined electrophysiological signs of diaphragmatic neuromuscular blockade; patients with myasthenia gravis, but not controls, exhibited a marked reduction in diaphragm action potentials with sustained phrenic stimulation. Our observation further supports the view that phrenic nerve stimulation can identify patients with neuromuscular transmission abnormalities who are at risk for respiratory failure. ${ }^{6}$ It could be very useful in the setting of drug induced neuromuscular disorders that often raise difficult diagnostic questions such as during recovery from anaesthesia.

\section{ROLE OF CIBENZOLINE}

Cibenzoline is a class Ic anti-arrhythmic agent with limited class III and class IV activity, which is pharmacologically close to quinidine. It is principally used in France and is not available in all countries.

Class Ic anti-arrhythmic agents interact with neuromuscular transmission at the presynaptic and post-synaptic levels ${ }^{8}$ and accidents similar to the one described here have been reported with procainamide and quinidine. ${ }^{12910}$ These substances can exacerbate myasthenia gravis or are responsible for myasthenia-like syndromes, with such typical features as generalised muscle weakness and bilateral ptosis, both of which were present in our patient. They have also been associated with acute myositis and signs suggestive of anticholinesterase poisoning. ${ }^{10-12}$ Fasciculations and a transient increase in the serum levels of creatine phosphokinase (617 and $1445 \mathrm{U} / \mathrm{ml}$ on days 7 and 8, respectively) suggest that this occurred to some degree in the present case.

There is no absolute proof that cibenzoline was responsible for the patient's respiratory failure. Indeed, because of the severity of the initial episode it was not considered ethical to propose a rechallenge, especially since it appeared likely that the neuromuscular blockade was caused by the very high serum levels of cibenzoline resulting from modified pharmacokinetics in renal insufficiency; ${ }^{13}$ a rechallenge at small doses that would not have resulted in relapse of neuromuscular blockade would not mandatorily have been conclusive. However, there are several reasons to suggest that cibenzoline was responsible for the respiratory failure. Firstly, the patient had no underlying disease other than chronic renal failure. The latter can be associated with phrenic nerve dysfunction, ${ }^{14}$ but the normal phrenic nerve latency and the absence of marked variations in renal function during the course of the episode argue against a significant contribution of chronic renal failure-associated phrenic nerve lesions to the respiratory disorders. The hypothesis of myasthenia gravis revealed by the acute episode and spontaneously resolving with time could also be raised. The rapid disappearance of myasthenic elements seems to argue against it, as does the absence of residual muscle weakness after six months. This hypothesis would not account for the elements that suggest associated myositis and an anticholinesterase-like effect namely, increased serum levels of creatine phosphokinase and fasciculations, respectively. Secondly, the recovery of diaphragm strength closely parallelled the fall in serum levels of cibenzoline. Thirdly, the patient received only cibenzoline and nifedipine at the time of respiratory failure. Nifedipine, which he had already received for several years, is not a likely candidate as the source of neuromuscular blockade but consideration must be given to its possible role in promoting or potentiating it. Indeed, the diaphragm may be particularly sensitive to the deleterious myoneural effects of calcium channel blockers ${ }^{15}$ because excitationcontraction coupling in this muscle is dependent on extracellular calcium. ${ }^{16}$ Although there do not seem to be data available to establish a dose-effect relationship for undesirable events associated with cibenzoline, such a potentiation could explain an apparent discrepancy between the serum level of cibenzoline (more than twice the upper limit of the normal range) and the magnitude of the observed effect on neuromuscular transmission.

In conclusion, our observation seems to confirm that drugs, or a combination of drugs, that possibly interfere with neuromuscular transmission can be a source of serious respiratory disorders, not only in patients with underlying neuromuscular or respiratory diseases but also in patients with a normal respiratory system who have an impaired ability to eliminate drugs.

The authors are grateful to Professor Benoit Wallaert for drawing the attention of our laboratory to the respiratory aspects of neuromuscular side effects of drugs.

1 Aldrich TK, Prezant DJ. Adverse effects of drugs on the respiratory muscles. Clin Chest Med 199011:177-89

Eriksson LI. Ventilation and neuromuscular blocking drugs. Acta Anaesthesiol Scand Suppl 1994;102:11-5.

3 Harron DW, Brogden RN, Faulds D, Fitton A. Cibenzoline. A review of its pharmacological properties and therapeutic potential in arrhythmias. Drugs 1992;43:734-59.

4 Hilleman DE, Mohiuddin SM, Ahmed IS, Dahl JM Cibenzoline-induced hypoglycemia. Drug Intell Clin Pharm 1987;21:38-40.

5 Delhez L. Modalités, chez l'homme normal, de la réponse électrique des piliers du diaphragme à la stimulation électrique des nerfs phréniques par des chocs uniques. Arch rique des nerfs phreniques par des
Int Physiol Biochim 1965;73:832-9.

6 Mier A, Brophy C, Moxham J, Green M. Repetitive stimu lation of phrenic nerves in myasthenia gravis. Thorax 1992, 47:640-4

7 Hamnegard CH, Wragg S, Kyroussis D, Mills G, Bake B, Green $\mathrm{M}$, et al. Mouth pressure in response to magnetic stimulation of the phrenic nerves. Thorax 1995;50:620-4

8 Lee DC, Kim YI, Liu HH, Johns TR. Presynaptic and postsynaptic actions of procainamide on neuromuscula transmission. Muscle Nerve 1983;6:442-7.

9 Drachman DA, Skom JH. Procainamide: a hazard in myasthenia gravis. Arch Neurol 1965;13:316-20.

10 Hall CD, Malouf N. Skeletal muscle weakness resulting from quinidine ingestion. South Med f 1987;80:403-4.

11 Fontiveros ES, Cumming WJ, Hudgson P. Procainamideinduced myositis. F Neurol Sci 1980;45:143-7.

2 Yagiela JA, Benoit PW. Skeletal-muscle damage from quinidine (letter). $N$ Engl f Med 1979;301:437.

13 Aronoff G, Brier M, Maver ML, Barbalas M, Aogaichi K, Sloan R, et al. Bioavailability and kinetics of cibenzoline in patients with normal and impaired renal function. 7 in patients with normal and im

14 Zifko U, Auinger M, Albrecht G, Kastenbauer T, Lahrmann $\mathrm{H}$, Grisold W, et al. Phrenic neuropathy in chronic renal failure. Thorax 1995;50:793-4

15 Zalman F, Perloff JK, Durant NN, Campion DS. Acute respiratory failure following intravenous verapamil in $\mathrm{Du}-$ chenne's muscular dystrophy. Am Heart f 1983;105:510-1

16 Aubier M, Viires N, Piquet J, Murciano D, Blanchet F, Marty C, et al. Effects of hypocalcemia on diaphragmatic strength generation. F Appl Physiol 1985;58:2054-61. 